\title{
Management of Anorectal Malformation: Experience from Ethiopia
}

\author{
Maru Gama ${ }^{1}$, Amezene Tadesse ${ }^{2}$ \\ 1. Saint Paul Millennium Medical College \\ 2. School of Medicine, Addis Ababa University
}

Correspondence to: Dr.Amezene Tadesse, P.O Box 9086, Black Lion Hospital, Addis Ababa, Ethiopia. Email: amezenet@yahoo.com

\begin{abstract}
Background: Anorectal malformations are among the most common congenital malformation seen in children. There is paucity of data regarding the incidence and treatment outcome of this disease from Ethiopia. Methods: This is a retrospective review of patients treated for ARM at the TASH from March 2010-February 2014. Relevant statistical analysis was done and the results presented in tables and graphs.
\end{abstract} Results: Six patients $(6 \%)$ presented at early neonatal age (before the age of 4 days) and 14 (14\%) presented between 4 days and one month. A total of 57 associated congenital malformations were diagnosed in 44/99(44\%) patients. The two most common type of ARM diagnosed among the female patients were rectovestibular fistula which occurred in 39/56 (70\%) and

\section{Introduction}

Anorectal malformations comprise a wide spectrum of anomalies of the anorectal system, urogenital system, sacral spine and perineal musculature (1). The extent of anomalies in these four components decides the type and severity of malformation (2). Anorectal malformations are among the most common congenital malformation seen in children (1-3). The incidence worldwide varies from 1 in 1500 to 1 in 5000(3). In one study conducted in Cape Town, the incidence is reported as 1:1740 among whites, 1:1770 among colored and 1: 2260 among blacks (4). ARMs may be classified according to the Krickenbeck's international classification which helps to do uniform reporting and comparison in males and females (5). This is done according to the number of orifices seen in the perineum for females and the location of fistula in males (5). Associated malformations are significant both epidemiologically and prognostically in terms of perineal fistula accounting for 6/56 (11\%). ARM with urethral fistula was the commonest diagnosis among the male patients, making up 10/43 (23\%). Post-operative outcome showed that $64(64 \%)$ had a very good functional outcome. Conclusions: The diagnosis of anorectal malformation is late. Nearly half of the patients were found to have associated malformations

Key words: Anorectal malformation, Associated malformations, Postero-sagittal anorectoplasty

Ann Afr Surg. 2018; 15(1):25-28

DOI:http://dx.doi.org/10.4314/aas.v15i1.6

(C) 2018 Author. This work is licensed under the Creative Commons Attribution 4.0 International License

morbidity and mortality. These malformations are present in 45-65\% of neonates with ARMs (6). These congenital malformations include urogenital, cardiac, vertebral, spinal and gastrointestinal abnormalities in the majority of the cases (6). The management of ARM ranges from classical Postero-sagittal anorectoplasty (PSARP) to minimal invasive procedures such as minimal anoplasty. The aim of the surgical management is to reconstruct the rectum/anus, genital tract and the urinary tract. Normal sexual function and the potential to bear children later in life are also very important long term outcome (3). However, the fecal and urinary incontinence may persist even after an excellent anatomic repair, mainly due to associated congenital problems (7). Globally, $75 \%$ of all patients have voluntary bowel movement after repair, half of these still soil their under wear. Constipation is also a major common sequel (1). There is paucity of data regarding 
the incidence and treatment outcome of this disease from Ethiopia. To our knowledge, there is only one article published more than 36 years back from Ethiopia (8). Since then, there has been a lot of change about the understanding, classification, management and outcome of the ARM. The objective of this study is to describe the pattern of patients treated for ARM at the Tikur Anbessa Specialized Hospital (TASH), Addis Ababa, Ethiopia, with special emphasis on the presentation, management and outcome of children with ARM and its associated malformations. TASH is a tertiary teaching referral hospital for the whole of the nation with a population of 100 million of which $41.5 \%$ are under 15 years old. It has 40 pediatric surgery beds and annually it operates around 1000 pediatric surgery cases.

\section{Methods}

This is a retrospective review of patients treated for ARM at the TASH from March 2010-February 2014. Data was collected from the operation theatre logbook and individual patient's records. During the study period 112 patients were operated, of which only the medical records of 99 patients retrieved and analyzed. The collected data consisted of the patients' sociodemographic profiles, presenting symptoms, physical findings, investigations performed, specific diagnosis, surgical interventions performed including emergency and definitive procedures, presence of associated malformations and outcomes.

Delay in presentation was defined as those cases who presented after 72 hours of delivery. The definitive procedures include minimal anoplasty, antero-saggittal anorectoplasty (ASARP) and PSARP. ASARP was used in female patients with recto vestibular fistula. PSARP is reserved for all other anomalies except those who can be managed by minimal anoplasty.

Data was collected by using a pre-structured questionnaire, and analysis done using SPSS version 20 software. Relevant statistical analysis was done and the results presented in tables and pie chart. Statistical analysis was made for selected variables.

\section{Results}

A total of 99 patients were seen and treated at the TASH for ARM during the study period. All the patients' charts were retrieved and hence were included in the study. Forty-three of the patients were males and The ANNALS of AFRICAN SURGERY, January 2018 Volume 15 Issue 1 26 the rest 56 were females, making the male to female ratio $0.76: 1$. Only six $(6 \%)$ presented at early neonatal age (before the age of 4 days) and 14 (14\%) presented between 4 days and one month). Eleven (11\%) patients presented after 5 years of age.

\section{Table 1: Age and sex distribution}

\begin{tabular}{|l|l|l|l|}
\hline AGE & $\begin{array}{l}\text { Male } \\
(\mathrm{N}=43)\end{array}$ & $\begin{array}{l}\text { Female } \\
(\mathrm{N}=56)\end{array}$ & TOTAL \\
\hline Birth -3 days & 5 & 1 & $6(6 \%)$ \\
\hline 4 days -30 days & 10 & 4 & $14(14 \%)$ \\
\hline 31 days- 12 months & 12 & 22 & $34(34 \%)$ \\
\hline 1 year - 5 years & 12 & 22 & $34(34 \%)$ \\
\hline 5 years -14 years & 4 & 7 & $11(11 \%)$ \\
\hline TOTAL & 43 & 56 & $99(100 \%)$ \\
\hline
\end{tabular}

Most patients (46\%) presented with passage of meconium or fecal matter through abnormal routes without significant intestinal obstruction. Features of intestinal obstruction without any sign of fistula was seen in $40(40 \%)$ of the patients. Intestinal obstruction in addition to passage of fecal matter through abnormal routes was seen in $13(13 \%)$

While Fifty-five patients presented with isolated ARM, 57 associated congenital malformations were diagnosed in 44/99(44\%) patients. The commonest anomalies were genitourinary (undescended testis (UDT) 8 , hypospadias 6 , ectopic kidneys 6 , renal agenesis 4 , vesico-ureteric reflux (VUR) 3, duplicated systems 3 and renal fusion 1). Four patients with ARM has 3 associated lesions with the following combination (trachea-esophageal fistula (TEF), spina bifida and UDT; renal agenesis, VUR and intestinal atresia; ectopic kidney, UDT and cardiac anomaly; hypospadias, UDT and spina bifida). Five ARM patients have 2 associated lesions [UDT and TEF; VUR and cardiac anomaly; spina bifida and hypospadias; TEF and renal agenesis; VUR and spina bifida]. Thirty-five patients presented with a single associated anomaly (Table2).

Table 2: Associated Malformations

\begin{tabular}{|l|l|}
\hline Associated Malformation & $\mathrm{N}(\%)$ \\
\hline Genitourinary Malformation 31 & $31(54)$ \\
\hline Spina bifida 10 & $10(18)$ \\
\hline Cardiac anomaly 7 & $7(12)$ \\
\hline Esophageal atresia with Tracheo-esophageal fistula & $5(9)$ \\
\hline Intestinal atresia 4 & $4(7)$ \\
\hline
\end{tabular}


The two most common type of ARM diagnosed among the female patients were recto-vestibular fistula which occurred in 39/56 (70\%) and perineal fistula accounting for $6 / 56(11 \%)$. Three (5.4\%) did not have fistula (Table $3)$.

Table 3: Types of ARM in female patients

\begin{tabular}{|l|l|l|}
\hline Type of ARM & $\mathrm{N}=56$ & $\%$ \\
\hline Recto vestibular fistula & 39 & 70 \\
\hline Perineal (cutaneous) fistula & 6 & 11 \\
\hline Cloaca & 4 & 7 \\
\hline ARM Without fistula & 3 & 5 \\
\hline Recto vaginal fistula & 3 & 5 \\
\hline Anal stenosis & 1 & 2 \\
\hline
\end{tabular}

ARM with urethral fistula was the commonest diagnosis among the male patients, making up 10/43 (23\%), followed by ARM without fistula 9/43(21\%) and covered anus $8 / 43(19 \%)$. The variant of the urethral fistula couldn't be specified either because distal colostogram couldn't be done or the contrast study was not done properly (Table 4).

\section{Table 4: Types of ARM in Male Patients}

\begin{tabular}{|l|l|l|}
\hline Types of ARM & $\mathrm{N}=43$ & $\%$ \\
\hline Recto urethral fistula & 10 & 23 \\
\hline ARM without fistula & 9 & 21 \\
\hline Covered anus & 8 & 19 \\
\hline Perineal (cutaneous) fistula & 6 & 14 \\
\hline Recto-vesical fistula & 5 & 12 \\
\hline ARM With bucket handle & 4 & 9 \\
\hline Anal stenosis & 1 & 2 \\
\hline Total number & 43 & 100 \\
\hline
\end{tabular}

Colostomy was done for $95 \%(53 / 56)$ of female and $77 \%$ (33/43) of male patients either for the relief of obstruction or as the prerequisite for the definitive procedure. Minimal anoplasty is done for 5\% (3/56) of females and $23 \%(10 / 43)$ of male. PSARP was performed in $45 \%(45 / 99)$ while ASARP in $30 \%$ (30/99) female patients with recto-vestibular fistula. Nine female patients with recto-vestibular fistula were managed by PSARP. Twenty-one percent (21/99) of the patients underwent minimal anoplasty. Eight patients who were managed with colostomy on arrival were found to have low variant of ARM and were treated with minimal anoplasty. Three percent (3/99) did not appear for definitive management. Post operative outcome showed that $64(64 \%)$ had a very good functional outcome without a notable complication. However, $12(12 \%)$ developed anal stenosis with constipation and 4(4\%) developed anal soiling. Nine $(9 \%)$ were lost to follow up. (Table 5)

\section{Table 5: Outcomes}

\begin{tabular}{|l|l|}
\hline Outcome & $\mathrm{N}$ \\
\hline Very Good [normal] & 64 \\
\hline Stenosis with constipation & 12 \\
\hline Soiling & 4 \\
\hline Wound dehiscence & 4 \\
\hline Incontinence & 2 \\
\hline Recurrence of fistula & 1 \\
\hline Didn't Appear for follow up & 9 \\
\hline Others & 3 \\
\hline Total & 99 \\
\hline
\end{tabular}

\section{Discussion}

The number of patients treated during the five years of the study period is doubled when compared to the study conducted by Orvar Johnson and his colleagues in the same institution 36 years ago (8). This might be because of increased awareness and treatment seeking behavior of the population, increased access to service provider, improved referral system, increase population size or due to a combination of reasons which may need further study. However, if increased incidence of ARM really is the reason it has to be confirmed by other population based studies.

In this study, female patients outnumber the male counterparts slightly, $\mathrm{M}$ : $\mathrm{F}$ being $0.76: 1$. This finding is similar to the study done in Italy where $54 \%$ of their patients are females and to the proportion noticed from our institute 36 years back, 53\%:47\% respectively $(7,8)$. However, study done in South Africa indicated that the ratio of male to female patients is grossly equal $(4,6,9)$. There was a delay in presentation in $94 \%$ of the cases. Anorectal malformation is the most common cause of intestinal obstruction in the early neonatal period which was demonstrated in the study done in the same institute (10). However, it is noted that there is delay in presentation to our hospital. Though delayed diagnosis is seen in $27 \%$ of patients in study done in Italy and 
$24 \%$ of patients in Kosovo $(7,11)$, ours is significantly higher. All patients presented late are those cases with fistula such as Recto vestibular fistula, Perineal (cutaneous) fistula and anal stenosis in females and Perineal (cutaneous) fistula and anal stenosis in males. This very late presentation might be mainly because of the fact that these children do not develop acute intestinal obstruction due to the adequate size of the coexisting fistula. Lack of awareness of the treatability and lack of access to surgical service could be the reasons for the delay in presentation

Concerning the outcome of patients, 65\% (64/99) of our patients showed good (optimal) outcome. While $9 \%$ (9/99) patients never appeared for follow up after operation, $26 \%$ (26/99) of patients developed different complications post operatively. This is comparable to other studies that showed a complication rate of only $21 \%$ of complication (3).

Associated congenital malformation accounted for $44 \%$ (44/99) of patients. The large number of associated congenital malformation seen in this study is similar with the studies conducted in other studies $(2,6,7,13)$. Genitourinary malformations are the dominant associated lesions detected in this study which is $31 \%$ $(31 / 99)$ and it is in line with the studies done somewhere else $(2,11-13)$.

\section{Limitations}

We don't have standardized institutional screening protocol. Hence we might have missed some more patients with associated malformation.

\section{Conclusions}

The prevalence of ARM is almost similar among both sexes. The diagnosis of anorectal malformation in our setup is late. Nearly half of the patients were found to have associated malformations highlighting the importance of evaluation of neonates with ARM for associated malformations.

\section{References}

1. Pena A, Hong A. Advances in the Management of Anorectal Malformation. Am J Surg. 2000; 180(5):370-6.

2. Bhatnagar S. Anorectal Malformation. J Neonatal Surg. 2015; 4: 1.

3. Levitt MA, Pena A. Anorectal Malformations. Orphanet J Rare Dis. 2012; 7: 98.
4. Moore SW, Sidler D, Hadley GP. Anorectal Malformation in Africa. S Afr J Surg. 2005; 43 (4):174-5.

5. Gupta DK. Anorectal Malformations - Wingspread to Krickenbeck. J Indian Assoc Pediatr Surg. 2005; 10:75-7.

6. Thereon AP, Brisighelli G, Theron AE, et al. Comparison in the Incidence of Anorectal Malformation Between The First and Third World Referral Center. Pediatr Surg Int. 2015; 31(8):75964.

7. Gangopadhyay AN, Pandey V. Anorectal Malformations. J Indian Assoc Pediatr Surg. 2015; 20:10-5.

8. Rintala R, Midh L, Lindahl H. Fecal Continence and Quality of Life for Adult Patients with an Operated High or Intermediate Anorectal Malformation. J Pediatr Surg. 1994; 29(6):777-80.

9. Mohammed M, Amezene T, Tamirat M. Intestinal Obstruction in Early Neonatal Period: A 3-Year Review of Admitted Cases from a Tertiary Hospital in Ethiopia. J Health Sci. 2017; 27(4):393-400

10. Statovci S, Grajçevci S, Berisha M, et al. Late Diagnosis of Anorectal Malformations in Children. Surgical Science. 2015; 6:143-148.

11. Bhargava P, Mohajam JK, Kumar A. Anorectal Malformations in Children. J Indian Assoc Pediatr Surg. 2006; 11:3:136-9.

12. Cho S, Moore S, Fangman T. One Hundred Three Consecutive Patients with Anorectal Malformations and their Associated Malformation. Arch Pediatr Adolesc Med. 2001; 155:587-91. 